\title{
A self-help diet and physical activity intervention with dietetic support for weight management in men treated for prostate cancer: pilot study of the Prostate Cancer Weight Management (PRO-MAN) randomised controlled trial
}

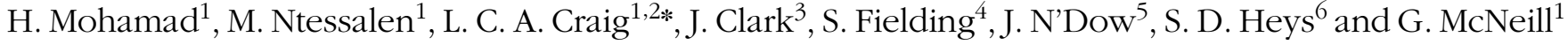 \\ ${ }^{1}$ Institute of Applied Health Sciences, University of Aberdeen, Foresterbill, Aberdeen AB25 2ZD, UK \\ ${ }^{2}$ Rowett Institute, University of Aberdeen, Foresterbill, Aberdeen AB25 2ZD, UK \\ ${ }^{3}$ NHS Grampian Department of Nutrition and Dietetics, Aberdeen, UK \\ ${ }^{4}$ Medical Statistics Team, Institute of Applied Health Sciences, University of Aberdeen, Foresterhill, Aberdeen AB25 2ZD, UK \\ ${ }^{5}$ Academic Urology Unit, Institute of Applied Health Sciences, University of Aberdeen, Foresterbill, Aberdeen AB25 2ZD, UK \\ ${ }^{6}$ School of Medicine, Medical Sciences and Nutrition, University of Aberdeen, Foresterbill, Aberdeen AB25 2ZD, UK
}

(Submitted 18 June 2018 - Final revision received 8 March 2019 - Accepted 31 March 2019)

\begin{abstract}
Overweight and obesity may increase risk of disease progression in men with prostate cancer, but there have been few studies of weight loss interventions in this patient group. In this study overweight or obese men treated for prostate cancer were randomised to a self-help diet and activity intervention with telephone-based dietitian support or a wait-list mini-intervention group. The intervention group had an initial group meeting, a supporting letter from their urological consultant, three telephone dietitian consultations at 4-week intervals, a pedometer and access to web-based diet and physical activity resources. At 12 weeks, men in both groups were given digital scales for providing follow-up weight measurements, and the wait-list group received a mini-intervention of the supporting letter, a pedometer and access to the web-based resources. Sixty-two men were randomised; fifty-four completed baseline and 12-week measurements, and fifty-one and twenty-seven provided measurements at 6 and 12 months, respectively. In a repeated-measures model, mean difference in weight change between groups (wait-list mini-intervention minus intervention) at 12 weeks was $-2 \cdot 13(95 \% \mathrm{CI}-3.44,-0 \cdot 82) \mathrm{kg}(P=0.002)$. At 12 months the corresponding value was $-2 \cdot 43(95 \%$ CI $-4.50,-0.37) \mathrm{kg}(P=0.022)$. Mean difference in global quality of life score change between groups at 12 weeks was $12.3(95 \%$ CI $4.93,19 \cdot 7)$ $(P=0.002)$; at 12 months there were no significant differences between groups. Results suggest the potential of self-help diet and physical activity intervention with trained support for modest but sustained weight loss in this patient group.
\end{abstract}

\section{Key words: Prostate cancer: Diet: Physical activity: Overweight: Obesity: Weight loss: Randomised controlled trials}

Prostate cancer is now the second-most common cancer in men worldwide: an estimated 1.1 million men were diagnosed with prostate cancer in 2012, accounting for $15 \%$ of all cancers in men ${ }^{(1)}$. In the UK, the lifetime risk of a diagnosis of prostate cancer for men is one in eight, with 47151 new cases in 2015 and 11631 deaths in $2016^{(2)}$. Excess body weight has been associated with an increased risk of diagnosis of more aggressive forms of prostate cancer and of recurrence and prostate cancer mortality ${ }^{(3-6)}$, although a recent umbrella review of the evidence suggests that these associations may be modest and the strength of evidence is 'suggestive' rather than 'convincing' ${ }^{\text {(7) }}$. One study found that weight gain following diagnosis was associated with an increased rate of prostate cancer-specific mortality ${ }^{(8)}$, suggesting that weight loss in those with excess weight could have beneficial outcomes, but to date there are no published studies on the influence of intentional weight loss on prostate cancer progression.

While weight loss can be achieved by energy restriction alone, physical activity may bring other health benefits such as reduced fatigue, increased muscle strength and improved cardio-metabolic health. For men with prostate cancer who receive androgen deprivation therapy, physical activity may help to prevent the loss of lean tissue mass ${ }^{(9)}$. One study of a 6-month diet and exercise intervention for men with prostate cancer receiving androgen deprivation therapy found beneficial effects on weight and fat mass as well as physical functioning ${ }^{(10)}$ but specific evidence-based physical activity guidelines for cancer survivors are lacking ${ }^{(11)}$. There is a lack of studies

Abbreviations: EORTC, European Organisation for Research and Treatment of Cancer; IQR, interquartile range; QoL, quality of life; UCAN, Urological Cancer.

* Corresponding author: Dr Leone Craig, email l.craig@abdn.ac.uk 
including physical activity combined with diet intervention for weight loss in prostate cancer patients, despite the evidence showing the additional benefits in terms of maintenance of lean tissue mass and other health benefits. From a systematic review of twenty randomised controlled trials of diet and exercise interventions in men treated for prostate cancer we concluded that low-fat or low-energy diets could lead to weight loss but that exercise could have other benefits such as improvement in quality of life $(\mathrm{QoL})^{(12)}$. This review revealed some gaps in the evidence including a lack of information on weight change in the trials of exercise and the optimum design of diet or exercise interventions which would encourage adherence in this group. Many of the studies combining diet and prescribed exercise have found poor retention and adherence rates, and a recent study by Focht et $\mathrm{al}$. suggested that a more personalised, self-directed weight loss intervention in prostate cancer patients rather than more supervised exercise and dietary advice showed promise for promoting adherence to independent lifestyle behaviour change ${ }^{(9)}$. To be applicable at scale, interventions need to be not only effective but also cost-effective, which supports the investigation of self-help resources such as internet and mobile-phone-delivered programmes. A systematic review of twenty-three studies of self-help interventions for weight loss found a mean difference in weight loss of 1.85 (95\% CI 0.83 , $2 \cdot 86) \mathrm{kg}$ at 6 months in favour of the intervention group though the difference was no longer significant at 12 months ${ }^{(13)}$. The same review found evidence of greater weight loss with more interactive programmes but less weight loss on self-help programmes in more socio-economically disadvantaged groups. The Reach Out to Enhance Wellness (RENEW) study of a home-based diet and exercise intervention in overweight or obese survivors of colorectal, breast and prostate cancer survivors aged over 65 years in the USA found weight loss and preservation of physical function following the intervention ${ }^{(14)}$. In this paper, we report the results of a pilot randomised controlled trial of a 12-week self-help diet and physical activity intervention on body weight and QoL in overweight and obese men treated for prostate cancer.

\section{Methods}

The Prostate Cancer Weight Management (PRO-MAN) study was conducted in North-East Scotland between October 2013 and April 2015. Recruitment was carried out through the Urological Cancer (UCAN) database, which covers all urology cancer patients in Grampian, Orkney and Shetland regions.

\section{Intervention design}

To tailor the intervention to the needs and preferences of men with prostate cancer we carried out a questionnaire survey in 265 men. Thirty-four of these men also took part in focus group discussions, with fourteen men being accompanied by their partners. The questionnaire results indicated that the majority (58\%) would prefer to do exercise on their own rather than in a group, with walking, cycling and swimming being considered suitable forms of exercise for 79,35 and $25 \%$ of men respectively. For the majority of respondents the partners carried out most of the food purchasing (62\%) and preparation (66\%); in the focus group discussions it emerged that these men and their partners would like information on specific foods to eat or avoid and control of appetite and portion size, ideally delivered in a one-to-one rather than group setting $(\mathrm{H}$ Mohamad, $\mathrm{PhD}$ thesis, University of Aberdeen).

Based on this information and literature which suggested that support from a clinician can promote adherence to behaviour change programmes ${ }^{(15)}$ we designed a self-help intervention package which included an initial group meeting, a letter of recommendation from the hospital consultant, a pedometer, telephone-based diet advice and access to online diet and physical activity resources. At the beginning of the 12-week programme, there was a $1 \mathrm{~h}$ group session with the two dietitians (H. M. and J. C.) to give an overview of the study including the duration of the study and frequency of contact and a demonstration of how to access the web-based self-help resources. Three groups were held in the evening at the CLAN local cancer support charity centre, and one group was held in the UCAN centre at Aberdeen Royal Infirmary during the daytime. Each participant was given a pedometer as a self-monitoring tool to encourage walking according to his own personal goals and a letter from their individual urological consultant to encourage them to comply with dietary modification and to engage in regular physical activity. The letter included the link and password to the web-based self-help resources in the UCAN website which provided additional written resources on appropriate diet and physical activity such as World Cancer Research Fund and other advice sheets and recipes. All participants and their partners where requested received their own username and password to access the resources.

Within a week of baseline measurement, each participant was contacted by one of the dietitians. A $24 \mathrm{~h}$ diet recall was undertaken to facilitate discussion around current food and drink intake and to allow the dietitian to give individually tailored dietary advice. The advice related to energy reduction through decreasing portion sizes, reducing high-energy, high-fat, highsugar foods, reducing alcohol and encouraging higher consumption of fruits, vegetables and wholegrains. Physical activity advice was based on individual capability and preferences, based on the discussion and self-monitoring of walking from the pedometer. Participants were asked to provide a pedometer reading at the two follow-up telephone calls to guide the advice provided.

At the end of the telephone call, each participant set personal diet and activity goals for the following 4 weeks, in discussion with the dietitian. A written summary of the goals set was sent out by mail to each participant. Following the initial phone call, two further calls were made at 4 and 8 weeks follow-up. These calls reviewed the goals and re-set new goals if required. The telephone calls were recorded and used to summarise the goals set.

\section{Participants}

Recruitment was carried out between October and December 2013. Inclusion criteria were age 16 years or over, a diagnosis of localised or locally advanced prostate cancer within the last 
36 months and overweight or obesity, which was defined as BMI $\geq 25 \mathrm{~kg} / \mathrm{m}^{2}$. In men aged 70 years or over only those with BMI $\geq 30 \mathrm{~kg} / \mathrm{m}^{2}$ were included to avoid adverse effects of weight loss on mortality reported in overweight adults in this age group $^{(16)}$. Exclusion criteria were evidence of distant metastases or current involvement with any weight management programme or other research studies. Potentially eligible participants were selected from the UCAN database. As the UCAN database did not contain information on BMI, men who were eligible on the basis of age and clinical stage were sent a letter of invitation to participate in the study and a reply slip which also asked for self-reported height and weight. Those who met the BMI inclusion criteria on the basis of self-reported height and weight were invited to attend a baseline meeting at which height and weight were measured by one of the dietitians. Those who met the BMI criteria on the basis of measured height and weight and who agreed to participate were randomised to either intervention or wait-list mini-intervention group using a minimisation programme based on age, time since diagnosis and BMI.

\section{Intervention group}

Men in the intervention group received all components of the intervention described earlier. At 12 weeks, they attended individual appointments with one of the dietitians in the UCAN centre at which weight was measured.

\section{Wait-list mini-intervention group}

Men in the wait-list mini-intervention group were seen individually at the UCAN centre for measurement of baseline height and weight. They were not given any instruction on diet and physical activity at this point but were asked to attend a second meeting at the UCAN centre at 12 weeks, at which point weight was measured and they were provided with a pedometer, a recommendation letter from their consultant and a password for the web-based self-help resources.

\section{Measurement of outcomes}

The primary outcomes were differences between groups in change in body weight at 12 weeks and 12 months, which was measured to the nearest $0 \cdot 1 \mathrm{~kg}$ using calibrated digital scales (SECA, model 803). At baseline and 12 weeks, the measurements were made by one of the dietitians. At the 12 week meeting, each participant was given a set of the same digital weighing scales along with written instructions on how to take measurements of weight at later time points. Around 6 and 12 months, a record sheet for providing weight measurement was mailed to each participant.

The secondary outcome was health-related QoL which was measured at baseline, 12 weeks and 6 and 12 months using the European Organisation for Research and Treatment of Cancer (EORTC) QLQ-C30 for assessing the generic aspects of QOL, together with EORTC QLQ-PR25 which is specific for prostate cancer ${ }^{(17)}$. The questionnaire was mailed to the participants before the baseline and 12 week meetings and with the follow-up weight record sheet at around 6 and 12 months along with a FREEPOST envelope to return the completed questionnaire. All of the scales and single-item measures were linearly transformed to a $0-100$ score using the scoring procedure described by Fayers et $a l^{(18)}$.

The feasibility and acceptability of the intervention were assessed using a questionnaire and auto-generated data on website use. The questionnaire collected information on participants' views of the setting, content and delivery of the intervention, using a five-point response scale to statements from strongly agree to strongly disagree, with an open text box at the end for any other comments. Participants in the intervention group completed this at the end of the 12 weeks and the wait-list mini-intervention group at the 6 month follow-up. Website use was tracked from baseline to 12 weeks for the intervention and 12 weeks to 6 months in the wait-list mini-intervention group.

\section{Data analysis}

Baseline characteristics were summarised as means and standard deviations or medians and interquartile ranges (IQR) in each randomised group and overall. Analysis of the repeated measures of weight and QoL up to 12 months was carried out using SAS, version 9.3 (SAS Institute Inc.). A linear mixed model with unstructured covariance matrix was fitted to assess fixed effects of time, group and time $\times$ group, adjusting for BMI, age at baseline and years since diagnosis. All tests were twotailed, and an $\alpha$ level of 0.05 was applied as the criterion for statistical significance. Estimates of difference in weight change and the $95 \% \mathrm{CI}$ are reported from the mixed model for each treatment group (baseline to 12 weeks, baseline to 6 months and baseline to 12 months). In addition the difference between the treatment groups is presented. All men with baseline measurement were included in the analysis as the mixed-model approach allows for missing data as long as one observed outcome (at any follow-up) is available. An intention-to-treat analysis was conducted, with men included in the group as randomised ( $n$ 54).

\section{Statistical power}

As sample size was limited by feasibility of recruitment, the minimal effect size was calculated. For weight change at 12 weeks the minimal detectable difference between the two groups ( $n 26$ and $n$ 28) with $90 \%$ power at the $5 \%$ significance level was $2.24 \mathrm{~kg}$ (assuming $\mathrm{SD}=2 \cdot 49$ ), and for weight change at 12 months the minimum detectable difference between the two groups was $5.56 \mathrm{~kg}$ (assuming SD $=4 \cdot 21, n 16$ and $n$ 11).

\section{Ethical committee approval}

The present study was conducted according to the guidelines laid down in the Declaration of Helsinki, and all procedures involving human subjects were approved by the North of Scotland Research Ethics Service. The initial study protocol, which included measurements up to 6 months, was approved by the North of Scotland Research Ethics Service on 9 August 2013 (REC reference 12/NS/0126) and subsequently approved by NHS R\&D on 16 September 2013 (reference 2012ON019). A study amendment to allow postal follow-up at 12 months was approved by the North of Scotland Research Ethics 


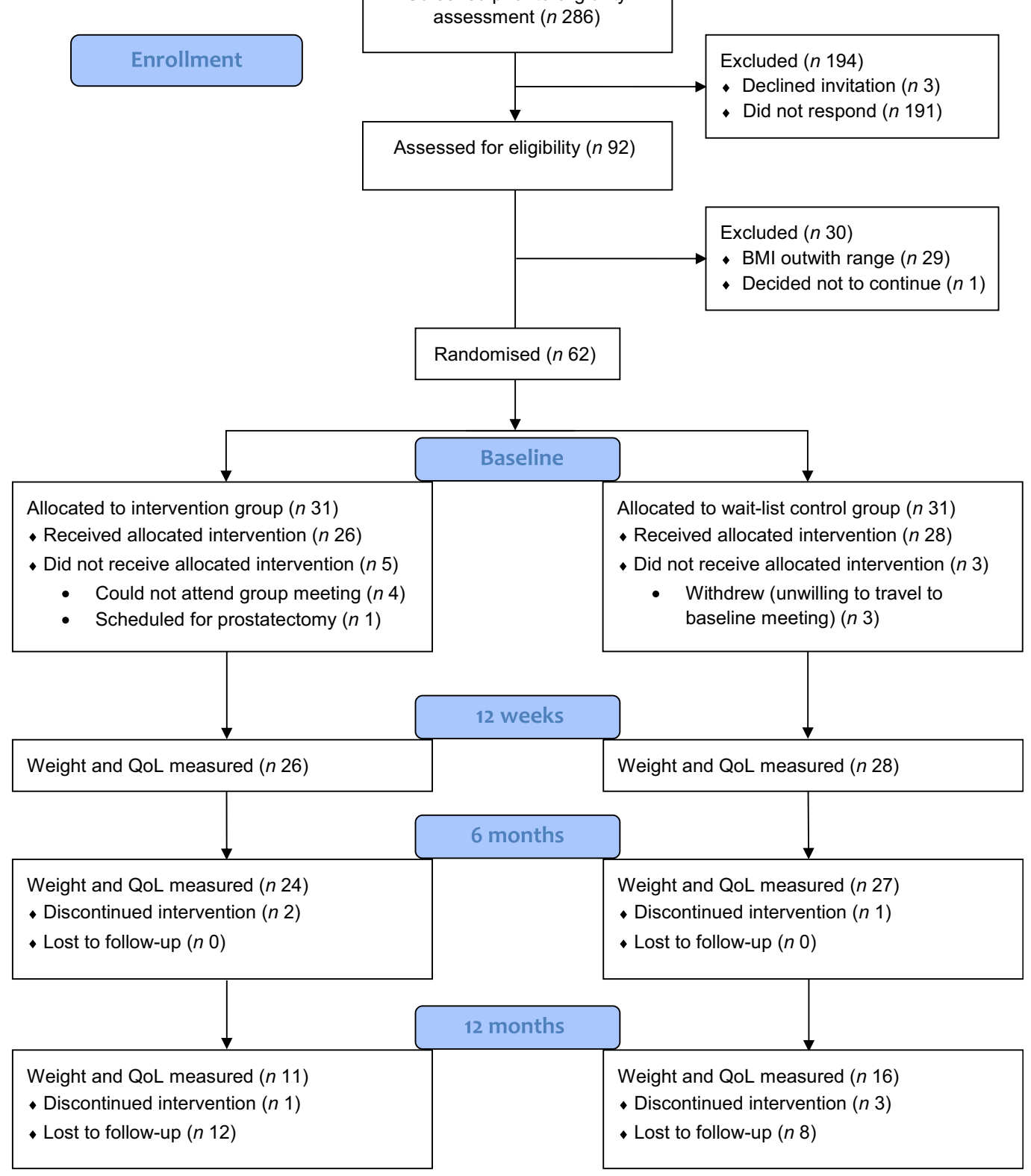

Fig. 1. Consolidated Standards of Reporting Trials (CONSORT) diagram of study recruitment and retention. QoL, quality of life.

Service on 20 February 2015. Written informed consent was obtained from all subjects.

\section{Trial registration}

The study was registered in Current Controlled Trials; registration number ISRCTN46025196, https://doi.org/10.1186/ ISRCTN46025196.

\section{Results}

\section{Recruitment and retention}

Of the 313 men on the UCAN database, 286 potentially suitable participants were approached. Ninety-two (32\%) expressed an interest in participation, 3 (1\%) declined participation and 191 (67\%) did not respond (Fig. 1). Of those who expressed an interest in the study, twenty-nine were below the minimum BMI according to their age group and one man decided not to continue due to lack of time. The remaining sixty-two men were randomised to the intervention ( $n$ 31) and wait-list mini-intervention ( $n$ 31) groups. Four men randomised to the intervention group could not attend any of the group sessions, and another man withdrew as he was scheduled to undergo prostatectomy, leaving twenty-six men who attended for baseline measurements. Three men randomised to wait-list mini-intervention group were not willing to travel to the study location for baseline data collection as they lived too far away, leaving twenty-eight who attended for baseline measurements. 
Table 1. Physical and socio-demographic characteristics of participants at baseline (Mean values and standard deviations; medians and interquartile ranges (IQR))

\begin{tabular}{|c|c|c|c|c|c|c|}
\hline \multirow[b]{2}{*}{ Characteristics } & \multicolumn{2}{|c|}{ Total $(n 54)$} & \multicolumn{2}{|c|}{ Intervention (n 26) } & \multicolumn{2}{|c|}{$\begin{array}{l}\text { Wait-list mini-intervention } \\
\qquad(n \text { 28) }\end{array}$} \\
\hline & Mean & SD & Mean & SD & Mean & SD \\
\hline Age (years) & 65.5 & $5 \cdot 6$ & 65.5 & 4.7 & 65.4 & 6.4 \\
\hline Weight (kg) & 88.9 & $11 \cdot 7$ & $89 \cdot 6$ & $11 \cdot 8$ & $88 \cdot 2$ & 11.8 \\
\hline Height (m) & 1.73 & 0.07 & 1.73 & 0.07 & 1.73 & 0.07 \\
\hline BMI $\left(\mathrm{kg} / \mathrm{m}^{2}\right)$ & 29.6 & $2 \cdot 9$ & 29.8 & 3.1 & $29 \cdot 4$ & $2 \cdot 6$ \\
\hline \multicolumn{7}{|c|}{ Global QoL score } \\
\hline Median & \multirow{2}{*}{\multicolumn{2}{|c|}{$\begin{array}{c}83 \cdot 3 \\
66 \cdot 7,91 \cdot 7\end{array}$}} & \multirow{2}{*}{\multicolumn{2}{|c|}{$\begin{array}{c}79 \cdot 2 \\
66 \cdot 7,83 \cdot 3\end{array}$}} & \multirow{2}{*}{\multicolumn{2}{|c|}{$\begin{array}{c}83 \cdot 3 \\
66 \cdot 7,97.9\end{array}$}} \\
\hline IQR & & & & & & \\
\hline \multicolumn{7}{|c|}{ Functional QoL score } \\
\hline Median & \multirow{2}{*}{\multicolumn{2}{|c|}{$\begin{array}{c}91 \cdot 1 \\
79 \cdot 4,97 \cdot 8\end{array}$}} & \multirow{2}{*}{\multicolumn{2}{|c|}{$\begin{array}{c}91 \cdot 1 \\
81 \cdot 1,97 \cdot 8\end{array}$}} & \multirow{2}{*}{\multicolumn{2}{|c|}{$\begin{array}{c}93 \cdot 3 \\
76 \cdot 7,97 \cdot 8\end{array}$}} \\
\hline IQR & & & & & & \\
\hline \multicolumn{7}{|c|}{ Symptoms QoL score } \\
\hline Median & \multirow{2}{*}{\multicolumn{2}{|c|}{$\begin{array}{c}7 \cdot 7 \\
2 \cdot 6,16 \cdot 0\end{array}$}} & \multirow{2}{*}{\multicolumn{2}{|c|}{$\begin{array}{c}7 \cdot 7 \\
2 \cdot 6,10 \cdot 9\end{array}$}} & \multirow{2}{*}{\multicolumn{2}{|c|}{$\begin{array}{c}7 \cdot 7 \\
2 \cdot 6,21 \cdot 8\end{array}$}} \\
\hline IQR & & & & & & \\
\hline
\end{tabular}

QoL, quality of life.

Four group sessions were arranged for men in the intervention group. The group sessions consisted of four to nine participants; five partners also attended the groups. Following this meeting, the dietitians telephoned participants at 1, 4 and 8 weeks. Two men in the intervention group withdrew; one man changed his mind, and another man withdrew due to an unrelated health problem (coronary artery bypass). Twentyone participants completed all three planned telephone calls; three completed two out of three telephone calls due to work commitments and being away from home.

Twenty-four of the twenty-six men in the intervention group and all twenty-eight men in the wait-list mini-intervention group completed the outcome measurements at 12 weeks. At 6 months one participant from the wait-list mini-intervention group did not complete the weight and QoL measures as he had undergone a hip replacement. At 12 months, eleven men in the intervention group and sixteen in the wait-list mini-intervention group provided data on their weight and completed QoL questionnaires.

\section{Baseline characteristics}

The age range of participants was from 48 to 81 years with a mean of 65.5 (SD 5.6) years. Two-thirds were aged over 65 years. With regard to time since prostate cancer diagnosis, most (70 \%) had been diagnosed for more than a year. Mean weight, height and BMI were $88.9 \mathrm{~kg}, 1.73 \mathrm{~m}$ and $29.6 \mathrm{~kg} / \mathrm{m}^{2}$, respectively (Table 1). The groups were balanced at baseline.

QOL assessed by EORTC modules composed of functional scales, symptom scales and a global health status scale. The baseline median score for global QoL of the participants was 83.3 with the most high/good level of functioning in physical functioning and the lowest level of functioning in sexual activity.

\section{Change in weight}

The mean weight change in the intervention group at 12 weeks was $-1.89(95 \% \mathrm{CI}-2 \cdot 85,-0.93) \mathrm{kg}(P<0.001)$ and in the waitlist mini-intervention group it was $0.24(95 \% \mathrm{CI}-0 \cdot 65,1 \cdot 13) \mathrm{kg}$ $(P=0.592)$ which was significantly different from the intervention group $(P=0 \cdot 002)$ as shown in Table 2.
Fifty-one (twenty-four intervention and twenty-seven waitlist mini-intervention) and twenty-seven (eleven intervention and sixteen wait-list mini-intervention) men provided weight measurements at 6 and 12 months, respectively. Both intervention and wait-list mini-intervention groups lost a significant amount of weight between baseline and 6 months, but there was no significant difference in the change between baseline and 6 months between intervention and wait-list mini-intervention. At 12 months the average weight change was -3.75 (95\% CI -5.31, $-2.18) \mathrm{kg} ; P<0.001$ in the intervention group and -1.31 (95\% CI $-2.66,0.03) \mathrm{kg} ; P=0.055$ in the wait-list mini-intervention group, with a significantly greater change of on average $-2.43 \mathrm{~kg}$ $(P=0.022)$ in the intervention group.

Four of the eleven participants in the intervention group and one of the sixteen participants in the wait-list mini-intervention group achieved clinically important $\geq 5 \%$ weight loss at the 12 month follow-up.

\section{Change in quality of life}

The average global QoL score significantly increased in the intervention group and significantly decreased in the wait-list miniintervention group between baseline and 12 weeks. There was no significant change in global QoL in either group at 6 or 12 months. At both 12 weeks and 6 months there was a significant difference in the changes in global QoL scores between the two groups because the intervention group's scores improved whereas the wait-list mini-intervention group's scores decreased from the baseline measurements (Table 3).

There was no significant change in the symptoms QoL scale in either group at any of the time points. The functional QoL scale significantly improved in the intervention group between baseline and 12 weeks, but there was no change in the wait-list miniintervention group leading to a significant difference between the groups at this time point. However, there was no significant change in functional QoL in either group at 6 or 12 months.

\section{Adverse events}

No adverse events were reported during the intervention. 
Table 2. Estimates of weight change $(\mathrm{kg})$ from linear mixed effects model, adjusted for baseline age, BMl and time since diagnosis (Estimates and $95 \%$ confidence intervals)

\begin{tabular}{|c|c|c|c|}
\hline & Estimate & $95 \% \mathrm{Cl}$ & $P$ \\
\hline Mini-intervention: baseline to 12 weeks & 0.24 & $-0.65,1 \cdot 13$ & 0.592 \\
\hline Mini-intervention: baseline to 6 months & $-2 \cdot 12$ & $-3.44,-0.79$ & 0.002 \\
\hline Mini-intervention: baseline to 12 months & -1.31 & $-2 \cdot 66,0.03$ & 0.055 \\
\hline Intervention: baseline to 12 weeks & -1.89 & $-2 \cdot 85,-0.93$ & $<0.001$ \\
\hline Intervention: baseline to 6 months & -3.40 & $-4.81,-1.99$ & $<0.001$ \\
\hline Intervention: baseline to 12 months & -3.75 & $-5 \cdot 31,-2 \cdot 18$ & $<0.001$ \\
\hline Difference at 12 weeks (mini-intervention - intervention) & $-2 \cdot 13$ & $-3.44,-0.82$ & 0.002 \\
\hline Difference at 6 months (mini-intervention - intervention) & -1.28 & $-3.22,0.65$ & $0 \cdot 189$ \\
\hline Difference at 12 months (mini-intervention - intervention) & $-2 \cdot 43$ & $-4.50,-0.37$ & 0.022 \\
\hline
\end{tabular}

Table 3. Estimates of change in quality of life from linear mixed effects model, adjusted for baseline age, BMl and time since diagnosis (Estimates and $95 \%$ confidence intervals)

\begin{tabular}{|c|c|c|c|}
\hline & Estimate & $95 \% \mathrm{Cl}$ & $P$ \\
\hline \multicolumn{4}{|l|}{ Global } \\
\hline Mini-intervention: baseline to 12 weeks & $-5 \cdot 06$ & $-10 \cdot 1,-0.02$ & 0.049 \\
\hline Mini-intervention: baseline to 6 months & $-5 \cdot 14$ & $-10.5,0.26$ & 0.062 \\
\hline Mini-intervention: baseline to 12 months & -0.98 & $-7 \cdot 00,5 \cdot 04$ & 0.742 \\
\hline Intervention: baseline to 12 weeks & $7 \cdot 23$ & $-1 \cdot 86,12 \cdot 6$ & 0.009 \\
\hline Intervention: baseline to 6 months & 4.29 & $-1.42,10 \cdot 0$ & 0.137 \\
\hline Intervention: baseline to 12 months & -0.77 & $-7 \cdot 61,6.07$ & 0.821 \\
\hline Difference at 12 weeks (mini-intervention - intervention) & $12 \cdot 3$ & $4.93,19.7$ & 0.002 \\
\hline Difference at 6 months (mini-intervention - intervention) & 9.43 & $1.57,17.3$ & 0.020 \\
\hline Difference at 12 months (mini-intervention - intervention) & 0.22 & $-8 \cdot 89,9.33$ & 0.962 \\
\hline \multicolumn{4}{|l|}{ Functional } \\
\hline Mini-intervention: baseline to 12 weeks & $-1 \cdot 27$ & $-4 \cdot 55,2 \cdot 01$ & 0.440 \\
\hline Mini-intervention: baseline to 6 months & -1.72 & $-5 \cdot 51,2 \cdot 07$ & 0.367 \\
\hline Mini-intervention: baseline to 12 months & $2 \cdot 08$ & $-0.99,5 \cdot 16$ & 0.177 \\
\hline Intervention: baseline to 12 weeks & 3.97 & $0.44,7.50$ & 0.028 \\
\hline Intervention: baseline to 6 months & -0.21 & $-4.25,3.83$ & 0.916 \\
\hline Intervention: baseline to 12 months & $2 \cdot 65$ & $-0.85,6 \cdot 14$ & 0.133 \\
\hline Difference at 12 weeks (mini-intervention - intervention) & $5 \cdot 24$ & $0.42,10 \cdot 1$ & 0.033 \\
\hline Difference at 6 months (mini-intervention - intervention) & 1.50 & $-4.03,7.04$ & 0.588 \\
\hline Difference at 12 months (mini-intervention - intervention) & 0.56 & $-4 \cdot 08,5 \cdot 21$ & 0.807 \\
\hline \multicolumn{4}{|l|}{ Symptoms } \\
\hline Mini-intervention: baseline to 12 weeks & 0.73 & $-1 \cdot 11,2 \cdot 57$ & 0.429 \\
\hline Mini-intervention: baseline to 6 months & 1.00 & $-1 \cdot 38,3 \cdot 39$ & 0.399 \\
\hline Mini-intervention: baseline to 12 months & $-2 \cdot 46$ & $-5 \cdot 60,0.67$ & 0.118 \\
\hline Intervention: baseline to 12 weeks & -0.83 & $-2 \cdot 83,1 \cdot 16$ & 0.404 \\
\hline Intervention: baseline to 6 months & -0.03 & $-2 \cdot 55,2 \cdot 50$ & 0.983 \\
\hline Intervention: baseline to 12 months & -1.36 & $-4 \cdot 93,2 \cdot 22$ & 0.444 \\
\hline Difference at 12 weeks (mini-intervention - intervention) & -1.57 & $-4 \cdot 28,1 \cdot 15$ & 0.252 \\
\hline Difference at 6 months (mini-intervention - intervention) & -1.03 & $-4 \cdot 50,2 \cdot 44$ & 0.552 \\
\hline Difference at 12 months (mini-intervention - intervention) & $1 \cdot 11$ & $-3 \cdot 64,5 \cdot 85$ & 0.637 \\
\hline
\end{tabular}

\section{Website use}

Fifteen ( $58 \%$ ) of the twenty-four participants in the intervention group and thirteen ( $46 \%$ ) of the twenty-eight participants in the wait-list mini-intervention group accessed the online resources during the 12 weeks of monitoring. The median number of visits to the website was 5.0 (IQR 3.0, 15.0) for the intervention group and 8.0 (IQR $3.5,15.5$ ) for the wait-list mini-intervention. The median time spent on the website was $7 \cdot 7$ (IQR $1 \cdot 1,49.7$ ) $\mathrm{min}$ for the intervention group and 18.0 (IQR 8.4, 31.2) $\mathrm{min}$ for the wait-list mini-intervention group. There were no significant differences between the groups in either the median number of visits or in the time spent on the website.

\section{Discussion}

Research on weight management in men with prostate cancer is relatively new, and despite many potential benefits of lifestyle change in cancer survivors, this population is under-researched. Given the mean age of the patients with prostate cancer, tailored self-help programmes with low-intensity physical activity deserve investigation, because many may have side effects of treatments such as urinary incontinence as well as co-morbidities such as orthopaedic problems.

In the present study, we found relatively modest weight loss of $1.89 \mathrm{~kg}$ at 12 weeks in the intervention group, but by 12 months, the mean weight loss had increased to $3.75 \mathrm{~kg}$, while 
in the mini-intervention group mean weight loss at 12 months was $1.31 \mathrm{~kg}$. Two other community-based weight loss trials involving men in the UK reported fairly similar results. The Lighten Up trial in overweight and obese men and women recruited through primary care used eight different 12 -week programmes and found a range of mean weight loss (either objectively measured by the programme providers or researchers, or self-reported ( $40 \%$ )) from 1.37 to $4.43 \mathrm{~kg}$ at 12 weeks and 1.13 to $4.45 \mathrm{~kg}$ at 12 months, with greatest weight loss seen with commercial weight loss programmes ${ }^{(19)}$. The proportion of participants in each arm of the Lighten Up trial who achieved $5 \%$ weight loss at 12 months ranged from 14 to $31 \%$, comparable with $36 \%$ of the intervention arm participants in our study. The 12-week Football Fans in Training (FFIT) intervention in overweight and obese male football supporters in Scotland achieved greater weight loss of on average $5.80 \mathrm{~kg}$ at 12 weeks and $5.56 \mathrm{~kg}$ at 1 year ${ }^{(20)}$, but the intervention was more intensive as it involved weekly group exercise and lifestyle sessions lasting $90 \mathrm{~min}$, and weight was measured by the researchers. However, the proportion of participants who achieved $5 \%$ weight loss in the intervention arm was $39 \%$, comparable with $36 \%$ of the intervention arm in our study. These interventions were more intensive than that used in the present study: a better comparison is with data from a lifestyle intervention with motivational interviewing, telephone counselling and weighing scales in overweight and obese patients with colorectal adenoma in Scotland which reported weight loss measured by the researchers of $3.50 \mathrm{~kg}$ in the intervention group and a difference of $2.69 \mathrm{~kg}$ between intervention and control groups at 12 months ${ }^{(21)}$, similar to the corresponding values of 3.75 and $2.43 \mathrm{~kg}$, respectively, in the present study. The proportion of those in the intervention group who achieved $5 \%$ weight loss was $36 \%$ which was the same as in our study. The US-based RENEW study of self-directed weight management in overweight and obese survivors of breast, colorectal and prostate cancer, which involved mailed self-help materials and telephone counselling, reported a (self-reported) weight loss of approximately $2.46 \mathrm{~kg}$ in the intervention arms and $1.46 \mathrm{~kg}$ in the delayed intervention arms over the relevant 12 month intervention periods ${ }^{(14)}$.

In the present study, we found few consistent differences in QoL over the study. This may be a true finding or could reflect the fact that the EORTC questionnaire is more suited to patients undergoing active treatment than those who have largely recovered from any side effects of treatment and can lead more normal lives. Alternative measures of well-being could be explored in future studies.

A strength of the PRO-MAN intervention was the incorporation of the preferences of men treated for prostate cancer. The fact that walking was a realistic physical activity for the majority probably contributed to the popularity of the pedometers which allowed goal-setting and self-monitoring and may have increased adherence compared with higher intensity or gymbased exercise. Pedometers were also found to be popular and motivational for many participants in the Football Fans in Training trial ${ }^{(22)}$. Although men and their partners requested advice on specific foods to include or avoid, the fact that the telephone consultations and materials were able to focus on energy intake control and general healthy eating could indicate that reassurance that 'superfoods' were not specifically required for secondary prevention of cancer may have been helpful.

Another strength of the study was the low cost of the intervention package: the cost of the pedometer, dietitians' time and travel to the group meeting in the intervention group was approximately $£ 90$ per patient (€103). The provision of weighing scales added approximately $£ 30$ ( $€ 34$ ) per person to these costs: although the scales were provided for provision of weight data, it cannot be ruled out that they contributed to the longer-term results in both groups. The most expensive component of the intervention was the dietetic input for the initial group meeting and telephone support, but the superiority of the 12-month results in the intervention group suggests that this may have been important for sustained effects, consistent with the conclusions of a systematic review ${ }^{(13)}$. The least expensive components of the intervention were the consultant's letter and the web-based materials. Website usage data showed that the internet-based resources were not accessed frequently by the majority of participants, though this may have been because the materials were downloaded and subsequently used in print form.

The study also had some significant limitations. Only $32 \%$ of those invited to participate agreed to do so, though the lack of recent weight data for the men on the UCAN database meant that we could not exclude those who were not above the BMI cut-offs in the initial mailing and do not know how many men believed themselves to be normal weight and may not have responded to the invitation letter for this reason. Future studies should consider ways to obtain pre-recruitment weight and to tackle other factors which could contribute to low recruitment such as time and travel burden. The Grampian region has low population density beyond the city of Aberdeen, so providing group meetings at a wider range of locations and collecting follow-up data for example in general practice clinics could be considered. The fact that the wait-list mini-intervention group received a sub-set of the intervention package and were given this at 12 weeks makes it more difficult to compare results at 6 months, though at 12 months both groups had had no face-to-face or telephone contact since 12 weeks so the results at 12 months can be used to compare the different intervention packages. However, the 12 month results should be interpreted with caution as only twenty-seven men recorded data at 12 months, raising the possibility of selective loss to followup of those who had not lost weight subsequently overestimating the weight loss. The retention was much higher at 6 months which may reflect the fact that the 12-month follow-up was not part of the original protocol. Although we provided accurate scales and clear instructions for weighing to participants ensuring consistency of equipment and its use, the use of selfrecorded weight measurements at 6 and 12 months raises the possibility of reporting bias due to social desirability or demand characteristics, as participants may report lower weights leading to an overestimate of the weight loss. However, Demark-Wahnefried et al. ${ }^{(23)}$ found high levels of agreement between self-reported and clinically assessed BMI in cancer survivors aged 65 years and over and Jolly et $a l .{ }^{(19)}$ found that those who self-reported their weight had a smaller weight loss than those whose weight was objectively measured and therefore this did not appear to be 
overestimating weight loss. In addition, we did not record the energy intake or pedometer readings of participants; however, this may have changed the nature of and the response to the intervention by adding more self-monitoring. The study included all men with a diagnosis of localised or locally advanced prostate cancer within the past 36 months: we did not limit inclusion by stage or type of treatment but also did not record additional details about cancer stage, grade and treatments, and therefore apart from time since diagnosis we were unable to take clinical details into account in the analysis. Lack of these details, in particular of those taking hormonal therapy or who changed treatments over the course of the study, may have affected the outcomes as androgen deprivation therapy is known to affect body composition with increases in weight and body fat in parallel with decreases in muscle mass ${ }^{(9)}$. Conversely, active treatment of another form or cancer progression may have induced weight loss. In addition to affecting weight, QoL outcomes may have been influenced by treatment side effects or cancer progression. Finally, the measurement of outcome data and the statistical analysis was not blind to the allocation of participants. Future studies could consider alternative designs and outcome measures to overcome these issues.

The results suggest the potential of a self-help diet and physical activity intervention with trained support for modest but sustained weight loss in this patient group.

\section{Acknowledgements}

We thank the Urology consultants at Aberdeen Royal Infirmary and staff of the UCAN centre, especially Paul Mannion, for support with the conduct of the study and the CLAN centre staff for hosting intervention group meetings.

H. M. carried out this work while in receipt of a $\mathrm{PhD}$ scholarship from the Government of Malaysia. We thank Cancer Research in Aberdeen and NE Scotland (CRANES) for financial support for the pedometers and weighing scales. L. C. A. C. and G. McN. acknowledge support from the Rural and Environment Science and Analytical Services (RESAS) programme of the Scottish government.

H. M., J. C., L. C. A. C., J. N'D., S. D. H. and G. McN. contributed to the design of the initial study; H. M. and J. C. delivered the interventions and collected outcome data up to 6 months; M. N. designed and led the data collection at 12 months with support from L. C. A. C. and G. McN; S. F. carried out the statistical analysis; all authors read and critically reviewed the final manuscript which was drafted by H. M., L. C. A. C. and G. McN.

There are no conflicts of interest.

\section{References}

1. Ferlay J, Soerjomataram I, Ervik M, et al. (2013) GLOBOCAN 2012 v1.0, Prostate Cancer: Estimated Incidence, Mortality and Prevalence Worldwide in 2012. Lyon, France: International Agency for Research on Cancer. http://globocan. iarc.fr/Pages/fact_sheets_cancer.aspx (accessed June 2018).
2. Cancer Research UK (2018) Prostate cancer statistics. http:// www.cancerresearchuk.org/health-professional/cancerstatistics/statistics-by-cancer-type/prostate-cancer (accessed June 2018).

3. Bradbury BD, Wilk JB \& Kaye JA (2005) Obesity and the risk of prostate cancer (United States). Cancer Causes Control 16, 637-641.

4. Rodriguez C, Patel AV, Calle EE, et al. (2001) Body mass index, height, and prostate cancer mortality in two large cohorts of adult men in the United States. Cancer Epidemiol Biomarkers Prev 10, 345-353.

5. Stroup SP, Cullen J, Auge BK, et al. (2007) Effect of obesity on prostate-specific antigen recurrence after radiation therapy for localized prostate cancer as measured by the 2006 radiation therapy oncology group-American Society for Therapeutic Radiation and Oncology (RTOG-ASTRO) Phoenix consensus definition. Cancer 110, 1003-1009.

6. World Cancer Research Fund/American Institute for Cancer Research (2018) Continuous Update Project Expert Report. Diet, nutrition, physical activity, and prostate cancer. http:// dietandcancerreport.org (accessed June 2018).

7. Markozannes G, Tzoulaki I, Karli D, et al. (2016) Diet, body size, physical activity and risk of prostate cancer: an umbrella review of the evidence. Eur J Cancer 69, 61-69.

8. Rodriguez C, Freedland SJ, Deka A, et al. (2007) Body mass index, weight change, and risk of prostate cancer in the cancer prevention study II nutrition cohort. Cancer Epidemiol Biomarkers Prev 16, 63-69.

9. Focht CB, Lucas AR, Grainger E, et al. (2018) Effects of a groupmediated exercise and dietary intervention in the treatment of prostate cancer patients undergoing androgen deprivation therapy: results from the IDEA-P trial. Ann Behav Med 52, 412-428.

10. Bourke L, Doll H, Crank H, et al. (2011) Lifestyle intervention in men with advanced prostate cancer receiving androgen suppression therapy: a feasibility study. Cancer Epidemiol Biomarkers Prev 20, 647-657.

11. Buffart LM, Galvao DA, Brug J, et al. (2014) Evidence-based physical activity guidelines for cancer survivors: current guidelines, knowledge gaps and future research directions. Cancer Treat Rev 40, 327-340.

12. Mohamad H, McNeill G, Haseen F, et al. (2015) The effect of dietary and exercise interventions on body weight in prostate cancer patients: a systematic review. Nutr Cancer 67, 43-60.

13. Hartmann-Boyce J, Jebb SA, Fletcher R, et al. (2015) Self-help for weight loss in overweight and obese adults: systematic review and meta-analysis. Am J Publ Health 105, e43-e57.

14. Demark-Wahnefried W, Morey MC, Sloane R, et al. (2012) Reach Out to Enhance Wellness home-based diet-exercise intervention promotes reproducible and sustainable improvements in health behaviors, body weight and physical functioning on older overweight/obese cancer survivors. J Clin Oncol 30, 2354-2361.

15. Porter Starr KN \& Bales CW (2015) Excessive body weight in older adults: concerns and recommendations. Clin Geriatr Med 3, 311-326.

16. Satia JA, Walsh JF \& Pruthi RS (2009) Health behaviour changes in white and African American prostate cancer survivors. Cancer Nurs 32, 107-117.

17. Aaronson NK (1993) The European Organization for Research and Treatment of Cancer QLQ-C30: a quality-of-life instrument for use in international clinical trials in oncology. J Natl Cancer Inst 85, 365 .

18. Fayers $\mathrm{P}$, Aaronson NK, Bjordal K, et al. (2001) EORTC QLQC30 Scoring Manual, 3rd ed. Brussels: European Organisation for Research and Treatment of Cancer. 
19. Jolly K, Lewis A, Beach J, et al. (2011) Comparison of range of commercial or primary care led weight reduction programmes with minimal intervention control for weight loss in obesity: lighten up randomised controlled trial. BMJ 343, d6500.

20. Hunt K, Wyke S, Gray CM, et al. (2014) A gender-sensitised weight loss and healthy living programme for overweight and obese men delivered by Scottish Premier League football clubs (FFIT): a pragmatic randomised controlled trial. Lancet 383, 1211-1221.

21. Anderson AS, Craigie AM, Caswell S, et al. (2014) The impact of a bodyweight and physical activity intervention (BeWEL) initiated through a national colorectal cancer screening programme: randomised controlled trial. BMJ 348, g1823.

22. Donachie C, Wyke S, Mutrie N, et al. (2017) 'It's like a personal motivator that you carried around wi' you': utilising self-determination theory to understand men's experiences of using pedometers to increase physical activity in a weight management programme. Int J Behav Nutr Phys Act 14, 61.

23. Demark-Wahnefried W, Clipp EC, Morey MC, et al. (2006) Lifestyle intervention development study to improve physical function in older adults with cancer: outcomes from project LEAD. J Clin Oncol 24, 3465-3473. 